\title{
Prevalence of Chlamydia trachomatis and Other Bacterial Infections in Pelvic Inflammatory Disease - A Cross Sectional Study
}

\author{
Mirunalini Thondaiman ${ }^{1}$, Latha Chaturvedula ${ }^{2}$, Rakesh Singh ${ }^{3}$ \\ ${ }^{1,2}$ Department of Obstetrics and Gynaecology, Jawaharlal Institute of Postgraduate Medical \\ Education and Research, Gorimedu, Puducherry, India. ${ }^{3}$ Department of Microbiology, Jawaharlal \\ Institute of Postgraduate Medical Education and Research, Gorimedu, Puducherry, India.
}

\section{ABSTRACT}

\section{BACKGROUND}

Pelvic inflammatory disease is a spectrum of diseases affecting the female upper genital tract. It is a poly microbial infection and Chlamydia trachomatis is the most common causative agent. We have undertaken this study with the objective of determining the prevalence of Chlamydia trachomatis infection in pelvic inflammatory disease (PID).

\section{METHODS}

This cross-sectional study consisted of 100 non-pregnant women with clinical features of PID. Endocervical, vaginal swabs and blood samples were collected from them. Polymerase-chain-reaction test (PCR) and enzyme-linked immunosorbent assay (ELISA) for chlamydial detection and Gram's staining \& culture for other pathogens were performed. Chi-square and Fisher's exact tests were used to study association between the clinical features and Chlamydia trachomatis positivity.

\section{RESULTS}

The prevalence rate of chlamydia was $15.1 \%$. The other organisms isolated were candida species in $12.9 \%$ and Escherichia coli in $5.3 \%$ of the study population. There was no association between the various demographic variables, menstrual problems, fever, urinary symptoms, history of infertility, abortions, instrumentations and the presence of Chlamydia trachomatis. A significant association was found between the lower abdominal pain which increased during menstruation $(P=0.013)$ and the two clinical signs, cervical motion tenderness and uterine tenderness $(P=0.001)$ with the presence of Chlamydia trachomatis and other cervical infections.

\section{CONCLUSIONS}

The prevalence rate of Chlamydia trachomatis in pelvic inflammatory disease was 15 $\%$ and other organisms isolated from the cervical swabs were candida species and Escherichia coli.

\section{KEY WORDS}

Pelvic Inflammatory Diseases, Chlamydia trachomatis
Corresponding Author: Dr. Mirunalini Thondaiman, No. 17, 4th Cross, Ponniamman Nagar, Karayanchavadi, Poonamallee, Chennai, Tamil Nadu, India. E-mail:drtmirun@gmail.com

DOI: $10.14260 / \mathrm{jemds} / 2021 / 166$

How to Cite This Article:

Thondaiman M, Chaturvedula L, Singh $R$. Prevalence of chlamydia trachomatis and other bacterial infections in pelvic inflammatory disease - a cross sectional study. J Evolution Med Dent Sci 2021;10(11):773-778, DOI: 10.14260/jemds/2021/166

Submission 02-11-2020, Peer Review 24-12-2020, Acceptance 30-12-2020, Published 15-03-2021.

Copyright (c) 2021 Mirunalini Thondaiman et al. This is an open access article distributed under Creative Commons Attribution License [Attribution 4.0 International (CC BY 4.0)] 


\section{BACKGROUND}

Pelvic inflammatory disease (PID) is an infection affecting the female upper genital tract and is most common among the sexually active women of reproductive age group. ${ }^{1}$ The microorganisms from vagina or cervix ascend to the uterus, fallopian tubes or adjacent structures causing endometritis, salpingitis, tubo-ovarian abscess or peritonitis which can result in irreversible damage to these structures. PID if not treated can lead to serious long-term problems like chronic pelvic pain, infertility, ectopic pregnancy etc. Hence, a low threshold is recommended for initiating the empirical treatment in PID.

PID is a poly microbial infection with multiple microbial aetiologies like Chlamydia trachomatis, Neisseria gonorrhoea, candida species, trichomonas species, bacterial vaginosis, Staphylococcus aureus, streptococcus species, Escherichia coli, pseudomonas species, klebsiella species, proteus species, Gardnerella vaginalis, etc. Chlamydia trachomatis is one of the leading causes of pelvic inflammatory disease.

We have undertaken this study to determine the prevalence of Chlamydia trachomatis and other bacterial infections in pelvic inflammatory disease through cervical swab culture and serology.

\section{METHODS}

This cross-sectional study was conducted in the Department of Obstetrics \& Gynaecology in collaboration with the Department of Microbiology, JIPMER, Pondicherry from 2014 - 2016. The inclusion criteria were non-pregnant sexually active women more than 18 years of the age who attended Gynaecology OPD with any one of the following symptoms: lower abdominal pain, white discharge per vaginum, pain in upper right abdomen, abnormal menstrual bleeding, fever and chills, painful micturition, nausea, vomiting, dyspareunia and with presence of any one of the following signs of cervical motion tenderness, uterine tenderness or adnexal tenderness. Pregnant, post-menopausal, women who have undergone hysterectomy with or without bilateral salpingooophorectomy and women with gynaecological malignancies were excluded from the study.

\section{Brief Procedure}

A total of 100 women were recruited as per the inclusion criteria. After obtaining written informed consent, detailed history including age, demographic profile, symptomatology, menstrual history, history of promiscuity, prior history of PID, prior history of curettage, hysterolaparoscopy and induced abortions were recorded. In general examination, anaemia status, nourishment, body mass index (BMI) was noted. In bimanual examination, the clinical signs like forniceal tenderness, cervical motion tenderness and uterine tenderness were noted. 2 endocervical swabs for chlamydia detection with PCR, 2 endocervical and vaginal swabs for gonococcal and other organism's detection and $5 \mathrm{ml}$ of blood for the serological tests - IgG and IgM ELISA for chlamydia detection were collected from all the recruited population.

Presence of at least 4 - 5 epithelial cells per high power field in microscopy was established before storing the endocervical swab material at $-20^{\circ} \mathrm{C}$ for chlamydial PCR until use.

The endocervical and vaginal swabs were inoculated on blood agar, MacConkey's agar and chocolate agar media and endocervical swab was additionally inoculated on ThayerMartin agar medium. Identification of isolates was based on macroscopical examination of growth colonies, microscopical studies of isolates based on Gram's staining reaction and biochemical characterisation.

Yeast was identified by Gram's stain. Candida species was identified by conventional tests like germ tube test, morphology on corn meal agar, sugar fermentation and assimilation tests.

\section{Polymerase Chain Reaction (PCR) for Chlamydia}

DNA was extracted using QIAamp DNA extraction kit, which were then amplified and analysed by $2 \%$ agarose gel electrophoresis and illuminated via UV light in gel documentation machine. Chlamydia trachomatis infection was detected by the presence of $517 \mathrm{bp}$ band. Serum was separated and stored at $-20^{\circ} \mathrm{C}$ until use. It was used to detect IgG and IgM antibodies against Chlamydia trachomatis by ELISA kits.

\section{Statistical Analysis}

Chi-square and Fisher's exact tests were used to study associations between Chlamydia trachomatis positivity and socio-demographic, behavioural and clinical variables. A Pvalue of $<0.05$ was considered statistically significant. All data was analysed using the Statistical Package for Social Sciences (SPSS) version 20.0 PC software.

\section{RESULTS}

A total of ninety-three samples were analysed finally (7 samples were rejected due to technical faults). The mean age of women in our study was $31.9 \pm 5$ years (range 22 - 43 years), with PID being more prevalent in women greater than 30 years. Of the 93 women, 63 belonged to the rural and 30 to the urban population. The majority of the women had completed their secondary education amounting to $52.7 \%$ of the study population according to International Standard Classification of Education. On classifying women into different socioeconomic groups, the majority of women belonged to middle class accounting to around $70 \%$. Around $65 \%$ of the women had normal BMI. About $69 \%$ women had normal menstrual cycles, while $19.4 \%$ had oligomenorrhoea, $12 \%$ had polymenorrhoea.

\section{Symptomatology}

Excessive white discharge per vaginum was present in $37.6 \%$, foul- smelling discharge in $39.8 \%$ and itching in $45.2 \%$ of women. Around $63.4 \%$ of women had increased abdominal pain during menstruation, $85 \%$ had dyspareunia, $28 \%$ had increased frequency of micturition and $25.8 \%$ had dysuria. 
Out of the 11 patients who presented with fever, 5 women had high grade fever associated with chills and rigors. $18 \%$ of women had a recurrence of PID. $11.8 \%$ had primary infertility and $4.3 \%$ had secondary infertility. About $36.6 \%$ of women had a history of either one (31.2\%) or two (5.4\%) abortions. Out of 93 women, 38 women had undergone dilatation and curettage either for abortion or infertility workup and $10.8 \%$ of women had history of hysteroscopy. History of extramarital contact was present in $12.9 \%$ of women.

Among the study population, $60 \%$ of women were anaemic, $23 \%$ were diabetic and $5 \%$ were using immunosuppressant drugs (steroids) for conditions like bronchial asthma, arthritis etc.

\section{Clinical Signs}

Among the women in our study population almost all women had forniceal tenderness. Cervical motion tenderness was present in $87.1 \%$ and uterine tenderness in $36.6 \%$ of patients.

\section{Prevalence of Chlamydia in Women with PID}

In this study, women were considered to have chlamydial PID, when any one the three tests were positive. (1) Positive cervical swab chlamydial PCR, (2) Positive IgM in ELISA, (3) Positive IgG in ELISA. Among the women diagnosed to have PID clinically, $15.1 \%$ had Chlamydia trachomatis infection. Acute chlamydial infections identified with chlamydial PCR and IgM ELISA were $6.45 \%$ and chronic or recurrent chlamydial infections with IgG ELISA were $8.6 \%$. Escherichia coli were present in $5.3 \%$ and candida in $12.9 \%$ of the study population.

The organisms isolated from the vaginal secretions were candida species, Staphylococcus aureus, Escherichia coli and klebsiella species. Among the candidal infections, the common candidal species were C. glabrata (14) C. tropicalis (7) C. krusei., (2) C. albicans., (2) and C. parapsilosis (1)

Relationship of various demographic variables and prevalence of Chlamydia trachomatis and other bacterial infections in PID of the 93 women, $15 \%$ were tested positive for chlamydial infections, out of which $6.5 \%$ were present in 25 - 30 years age group and $5.4 \%$ in 30 - 35 years group. There was no significant association found between the age groups and presence of chlamydial infections or other cervical organisms in PID (the P-value was 0.25). No statistical difference was found between the prevalence of Chlamydia trachomatis or other cervical organisms and the residence status (urban and rural population), socioeconomic status and educational status.

Our study showed that chlamydial infection was present in $13 \%$ of the married women, $2.2 \%$ of the divorcees and the widows. Analysis showed no significant difference in the prevalence of chlamydia and other cervical infections and their marital status.

Chlamydia trachomatis was present in $5 \%$ of patients with oligomenorrhoea, $27 \%$ with polymenorrhoea, $21 \%$ with dysmenorrhoea, $25 \%$ with inter menstrual bleed and $27 \%$ with postcoital bleed. Non-chlamydial infections were present in $11 \%$ patients with oligomenorrhoea, $18 \%$ with polymenorrhoea, $19 \%$ with dysmenorrhoea, $12 \%$ with intermenstrual bleed and $18 \%$ with postcoital bleed. There was no significant relation between the prevalence of chlamydia or other cervical organism and menstrual cycle abnormalities.

All the patients included in this study had white discharge per vaginum and lower abdominal pain as one of their presenting complaints. We didn't find any significant relation between the prevalence of chlamydial and other cervical infections and the various characteristics of white discharge per vaginum like quantity, foul smell, itching etc.

\begin{tabular}{|cccc|}
\hline Pain Abdomen & $\begin{array}{c}\text { Chlamydial } \\
\text { Infections N = 14 } \\
\text { N (\%) }\end{array}$ & $\begin{array}{c}\text { Other Cervical } \\
\text { Organisms } \\
\mathbf{N}=17 \text { N (\%) }\end{array}$ & $\begin{array}{c}\text { Clinical PID } \\
\text { with Negative } \\
\text { Microbiology } \\
\text { N = 62 N (\%) }\end{array}$ \\
$\begin{array}{c}\text { Increased with } \\
\text { menstruation N = 59 }\end{array}$ & $11(11.8)$ & $15(16.1)$ & $33(35.4)$ \\
$\begin{array}{c}\text { No association with } \\
\text { menstruation N = } 34\end{array}$ & $3(3.2)$ & $2(2.2)$ & $29(31.1)$ \\
\hline $\begin{array}{c}\text { Table 1. Relationship of Lower Abdominal Pain with Menstruation } \\
\text { and Its Association with Prevalence of Chlamydia trachomatis }\end{array}$ \\
\hline \multicolumn{4}{|l}{ The P-value - 0.013 } \\
\hline
\end{tabular}

In this study, a significant association was noted between the lower abdominal pain which increased during menstruation with that of the prevalence of chlamydial and other non-chlamydial infections in PID, though lower abdominal pain is not specific to any particular organism.

Dyspareunia was one of the most common symptoms among the women with the clinical diagnosis of PID. Chlamydia trachomatis was present in $12.9 \%$ of women with dyspareunia, $15.3 \%$ of women with increased frequency of micturition and $12.5 \%$ of women with dysuria. But there was no association noted between dyspareunia and urinary symptoms and the prevalence of chlamydia and other cervical infections.

During our study period, 11 women presented with fever out of which 5 women had high-grade fever with chills and rigors. Of them, five had history of recent abortions and dilatation and curettage. One patient had undergone hysterosalpingography as a part of infertility work up. One patient had a recurrence of PID, where the previous episode had been treated with azithromycin. These patients were admitted and given inpatient treatment with ceftriaxone intravenous for a week till the acute episode subsided and discharged with doxycycline and metronidazole for 2 weeks. Chlamydia PCR and IgM ELISA was positive in 1 woman, IgG ELISA in 2 women.

Cervical swab culture was positive for Escherichia Coli in 3 women and candida species in 1 woman. Vaginal swab culture was positive for candida species in 5 women, Staphylococcus aureus in 2 women and Escherichia coli in 1 woman. The prevalence of Chlamydia trachomatis or other cervical infections and the fever had no statistically significant association.

In this study, $18.3 \%$ had a previous history of PID. In these women with recurrent PID, IgG ELISA for chlamydia was positive in 5 women and candida was present in one woman.

\begin{tabular}{|ccccc|}
\hline & $\begin{array}{c}\text { H / O Infertility } \\
\text { Present }\end{array}$ & $\begin{array}{c}\text { Primary } \\
\text { Infertility N = }\end{array}$ & $\begin{array}{c}\text { Secondary } \\
\text { Infertility N = 4 }\end{array}$ \\
$\begin{array}{c}\text { Chlamydia N = } 14 \\
\begin{array}{c}\text { Other cervical } \\
\text { infections N = 17 }\end{array}\end{array}$ & $3(3.2 \%)$ & $11(11.8 \%)$ & 0 & $3(3.2 \%)$ \\
\hline $\begin{array}{c}\text { Table 2. Relationship of Infertility and Prevalence of Chlamydia } \\
\text { trachomatis and Other Cervical Infections in PID }\end{array}$ & $12(12.9 \%)$ & $4(4.3 \%)$ & $1(1.1 \%)$ \\
\hline
\end{tabular}


Chlamydial infection was found to be common among women with secondary infertility than those with primary infertility, though it was not statistically significant. The prevalence of chlamydia and other cervical infections were quite similar in both nulliparous and multiparous women.

\begin{tabular}{|c|c|c|c|c|c|}
\hline & & $\begin{array}{l}\text { Chlamydial } \\
\text { Infections } \\
\mathrm{N}=14 \\
\mathrm{~N}(\%)\end{array}$ & $\begin{array}{c}\text { Other } \\
\text { Cervical } \\
\text { Organisms } \\
\mathrm{N}=17 \\
\mathrm{~N}(\%)\end{array}$ & $\begin{array}{l}\text { Clinical PID } \\
\text { with Negative } \\
\text { Microbiology } \\
\text { N = 62 N (\%) }\end{array}$ & $\begin{array}{c}\mathbf{P} \\
\text { Value }\end{array}$ \\
\hline \multirow{2}{*}{$\begin{array}{l}\text { History of } \\
\text { abortions }\end{array}$} & Absent $\mathrm{N}=59$ & $7(7.5)$ & $13(14)$ & 39 (41.9) & \multirow[b]{2}{*}{0.31} \\
\hline & $\begin{array}{c}\text { Present } \mathrm{N}= \\
34\end{array}$ & $7(7.5)$ & $4(4.3)$ & $23(24.7)$ & \\
\hline \multirow{2}{*}{$\begin{array}{l}\text { History of } \\
\text { dilatation } \\
\text { and } \\
\text { curettage }\end{array}$} & Absent $\mathrm{N}=55$ & $6(6.5)$ & 12 (12.9) & 37 (39.7) & \multirow[b]{2}{*}{0.29} \\
\hline & $\begin{array}{c}\text { Present } \mathrm{N}= \\
38\end{array}$ & $8(8.6)$ & $5(5.4)$ & $25(26.9)$ & \\
\hline \multirow{2}{*}{$\begin{array}{c}\text { History of } \\
\text { hysteroscopy }\end{array}$} & Absent $\mathrm{N}=82$ & $10(10.8)$ & $16(17.2)$ & $56(60.2)$ & \multirow[b]{2}{*}{0.10} \\
\hline & $\begin{array}{c}\text { Present } N= \\
11\end{array}$ & $4(4.3)$ & $1(1.1)$ & $6(6.5)$ & \\
\hline \multicolumn{6}{|c|}{$\begin{array}{c}\text { Table 3. Relationship between History of Abortions and } \\
\text { Instrumentations and Prevalence of Chlamydia trachomatis and } \\
\text { Other Cervical Infections in PID }\end{array}$} \\
\hline
\end{tabular}

In this study, no significant association was found between number of abortions, history of dilatation \& curettage and hysteroscopy and the prevalence of chlamydia and other cervical infections causing PID.

Out of the $12.9 \%$ of women with the history of an extramarital relationship, chlamydia was present in $1.1 \%$ and other cervical infections in $4.3 \%$. The history of an extramarital affair had no significant relationship to the prevalence of chlamydia and other cervical infections.

We have found that the prevalence of chlamydia and other cervical infections in PID are not related to the anaemia, obesity, diabetes or nutritional status.

\section{Clinical Signs}

Chlamydia was present in $15 \%$ of the women with forniceal and cervical motion tenderness and $10.8 \%$ of women with uterine tenderness. Similarly, other cervical infections like candida species, Escherichia coli were present in $18.2 \%$ of women with forniceal and cervical motion tenderness and 9.7 $\%$ of women with uterine tenderness.

\begin{tabular}{|c|c|c|c|}
\hline & $\begin{array}{c}\text { Chlamydial } \\
\text { Infections } \\
\mathrm{N}=14 \mathrm{~N}(\%)\end{array}$ & $\begin{array}{c}\text { Other } \\
\text { Cervical } \\
\text { Organisms } \\
\mathrm{N}=17 \mathrm{~N}(\%)\end{array}$ & $\begin{array}{c}\text { Clinical PID with } \\
\text { Negative } \\
\text { Microbiology } \\
\text { N = 62 N (\%) }\end{array}$ \\
\hline Uterine Absent $\mathrm{N}=59$ & $4(4.3)$ & $8(8.6)$ & $47(50.5)$ \\
\hline tenderness Present $\mathrm{N}=34$ & $10(10.8)$ & $9(9.7)$ & $15(16.1)$ \\
\hline \multicolumn{4}{|c|}{$\begin{array}{c}\text { Table 4. Relationship between the Uterine Tenderness and Prevalence } \\
\text { of Chlamydia trachomatis and Other Cervical Infections }\end{array}$} \\
\hline \multicolumn{4}{|c|}{ The P-value is 0.001} \\
\hline
\end{tabular}

Of the three clinical signs, uterine tenderness was significantly associated with the prevalence of Chlamydia trachomatis (P-value 0.000) and other non-chlamydial infections (P-value 0.01) in the clinically diagnosed PID.

\section{DISCUSSION}

The prevalence of chlamydial infection in this study was found to be $15.1 \%$. The prevalence of chlamydia in our study is comparable to that of the prevalence $(22.7 \%)$ noted in the study by Ravindran et al. ${ }^{1}$ Another study by Malhotra et al. in Delhi, 2008 reported the prevalence of chlamydia in PID as 23 $\%$ in women attending Gynaecology OPD and $19.9 \%$ in women attending STD OPD. ${ }^{2}$ The non-chlamydial organisms identified were Escherichia coli (5.3\%) and candida species (12.9\%). But the study by Mohapatra et al. found that the prevalence rate of chlamydia was $26.6 \%$, gonorrhoea $33 \%$, Staphylococcal aureus $15 \%$, streptococcus species $8 \%$, Escherichia coli $4 \%$, trichomonas species $3.3 \%$, candida species $6 \%$, mycobacteria species $4 \%$ and ureaplasma species $12 \% .^{3}$ The organisms detected in Spencer et al. study were streptococcus species (11 $\%)$, staphylococcus species (16\%), Escherichia coli (10\%), proteus species ( $3 \%$ ) and pseudomonas species (4\%). ${ }^{4}$ This difference in microbiology can be either because the sample size was very small to detect the prevalence of all bacterial infections or the prevalence of Neisseria gonorrhoea as the causative organism for PID has decreased, but the candidal infections were present in a significant number of patients. Hence it can be suggested that the empirical treatment for PID can include even an antifungal. A study in Tamilnadu also showed that genital chlamydial infections were present only in $3 \%$ and candida infections in $10 \% .^{5}$

The organisms isolated from the vaginal secretions were candida species, Staphylococcus aureus, Escherichia coli and klebsiella species. In a study by Mohapatra et al. organisms isolated from vaginal swabs were candida species $(9.4 \%)$, bacterial vaginosis $(7.8 \%)$, trichomonas species (1 \%), Neisseria gonorrhoea $(0.7 \%) .^{3}$

Nearly $87 \%$ of women in our study were between 25 - 40 years of age. Among the chlamydial positive PID, $50 \%$ of women were less than 30 years and $50 \%$ greater than 30 years of age. This is comparable with the study done by Ravindran et al. where most of the patients with PID (88.6\%) were within the $20-40$ years age group and $70 \%$ of the chlamydia positive PID patients were in the 20 - 40 years age group. ${ }^{1}$ Likewise in the study by Mohapatra et al., chlamydial infections were more prevalent in 20 - 30 years age group. ${ }^{3}$ Young people are more prone to PID as they are sexually very active, may have multiple sexual partners and increased host susceptibility as the chlamydial antibodies are in lower concentrations, due to larger cervical ectopy and increased permeability of cervical mucus in younger age women.

Around $70 \%$ of women with PID belong to middle class II and III of socioeconomic status. Chlamydia was present in 3.5 $\%$ of women belonging to class IV, $4.3 \%$ in class III, $5.4 \%$ in class II. In a study by Ravindran et al., $63.6 \%$ of PID cases were in the lower social classes IV and V and the majority (60\%) of the chlamydia positive patients belonged to the lower social classes IV and V. ${ }^{1}$ Increased prevalence of PID in the lower and middle socio-economic group may be due to the poor local hygiene and poor awareness about the prevention of sexually transmitted disease.

In our study chlamydial infections were present in $15 \%$ of the married women and $25 \%$ of the divorcees and widows. In the study by Ravindran et al., $17.5 \%$ of the married, $100 \%$ of widows and $50 \%$ of single women were tested positive for chlamydial infections. ${ }^{1}$ Likewise in a study by Simms et al. chlamydial infection was present in $8 \%$ of married women, 22 $\%$ of cohabiting women, $13 \%$ in widowed / separated / divorcee and $57 \%$ in unmarried women who were sexually active. ${ }^{6}$ Thus it can be concluded that the people in polygamous 
relationships have increased risk of acquiring chlamydial infections.

Chlamydia was present in $15.6 \%$ of women with normal cycles, $13.7 \%$ of women with menstrual cycle irregularities. In the study by Prathiba et al. the prevalence of chlamydia in patients with menstrual irregularities was studied, which showed a $75 \%$ prevalence rate of chlamydia. This contrary in results can be explained, because the aim of our study was to estimate the prevalence of chlamydia in women with PID but the study by Prathiba et al. estimated the prevalence of chlamydia in women with menstrual irregularities.

Chlamydia was present in $11 \%$ of women with dysmenorrhoea, $4 \%$ with intermenstrual bleed and $6 \%$ with postcoital bleed. This is concurrence with the study by Chacko et al. wherein $17 \%$ of the women presented with multiple symptoms like menstrual irregularities, vaginal discharge and pain abdomen. ${ }^{8}$

Chlamydia was present in about $23 \%$ of women with excessive vaginal discharge. This is in comparison with a study done by Ravindran et al., who reported chlamydial positivity in $40 \%$ of patients with foul-smelling vaginal discharge. ${ }^{1}$ In the study by Simms et al., chlamydial infection was present in $53.3 \%$ of patients with excessive vaginal discharge. ${ }^{6}$

Around $63.4 \%$ of our women reported increased abdominal pain during first 5 - 7 days of the menstrual cycle. Chlamydia was found in $19 \%$ of the women with such complaints. A significant association was found between the lower abdominal pain which increases during menstruation and the presence of chlamydial and other non-chlamydial infections in PID. This finding can be compared to that quoted in the study by Machado et al., where chlamydia was found in $10 \%$ of PID with increased abdominal pain during menstruation. ${ }^{9}$ This association can be explained by the fact that the peristaltic contractions of the uterus move fluid cephalad, facilitating the ascend of the organisms from the lower genital tract to uterus, fallopian tubes etc.

Dyspareunia was reported by $85 \%$ of women and chlamydia was positive in $15 \%$. This finding is in comparison to the study done by Ravindran et al. where dyspareunia was present in $72.7 \%$ of PID patients but chlamydia was positive in $20 \%{ }^{1}$

Chlamydia was present in $27 \%$ of women with urinary symptoms. Simms et al. reported that $35 \%$ of the PID patients presented with urinary symptoms. ${ }^{6}$ Urinary symptoms were common among women with PID because most of the organisms causing PID especially chlamydia can also cause urethritis.

Chlamydia was present in $27 \%$ of women with fever. In the study done by Ravindran et al., fever was present in $39 \%$ of the PID patients while none of the chlamydia positive patients had fever. ${ }^{1}$ In the study by Simms et al., fever was the presenting complaint in $47 \%$ of PID population. ${ }^{6}$

In our study, about $16 \%$ of women had history of infertility with secondary infertility being more common than primary infertility. Ravindran et al. reported that among the patients with PID $45.5 \%$ had subfertility and $20 \%$ had primary infertility. ${ }^{1}$ Secondary infertility is found to be more common than primary infertility in PID because there might be increased chances for the ascending infections to reach the upper genital tract following delivery or dilatation and curettage done for spontaneous or induced abortions.
About $36.6 \%$ of women with PID had history of either one or two abortions. History of dilatation and curettage was present in $40.9 \%$, history of hysteroscopy was noted in 10.8 $\%$ of women. During these procedures there is high chance for the lower genital tract infections to ascend to the endometrium and tubes causing PID. In a study by Simms et al., PID was present in $29 \%$ of women with history of abortions. ${ }^{6}$ In a study by Gogate et al., hysterosalpingography, dilatation and curettage (D \& C), laparotomy and laparoscopic procedures were significantly associated with PID.10

\section{Clinical Signs}

Almost all the women in our study population had forniceal tenderness on bimanual examination. Cervical motion tenderness was present in $87.1 \%$ and uterine tenderness in $36.6 \%$ of patients. Chlamydia was present in $15.05 \%$ of the women with forniceal and cervical motion tenderness and $10.8 \%$ of women with uterine tenderness. In the study by Simms et al., the pelvic organ tenderness on bimanual examination was present in $99 \%$ of the patients. ${ }^{6}$ Eggert et al. study showed that PID was diagnosed in $49 \%$ of women with both uterine and forniceal tenderness and in $20 \%$ with uterine tenderness alone. ${ }^{11}$

\section{CONCLUSIONS}

Prevalence rate of Chlamydia trachomatis in pelvic inflammatory disease was $15 \%$ and the other organisms isolated from the cervical swabs were candida species and Escherichia coli in the clinically diagnosed PID. Increase in lower abdominal pain during menstruation and uterine tenderness were significantly associated with the presence of chlamydial infections.

Data sharing statement provided by the authors is available with the full text of this article at jemds.com.

Financial or other competing interests: None.

Disclosure forms provided by the authors are available with the full text of this article at jemds.com.

\section{REFERENCES}

[1] Ravindran J, Tan YI, Ngeow YF. The prevalence of Chlamydia trachomatis in patients with pelvic inflammatory disease. Med J Malaysia 1998;53(1):16-21.

[2] Malhotra M, Bala M, Muralidhar S, et al. Prevalence of Chlamydia Trachomatis ans its association with other sexually transmitted infections in a tertiary care centre in North India. Indian J Sex Transm Dis 2008;29(2):82-5.

[3] Mohapatra S, Panda P, Parida B. Genital tract infection of women in Southern Orissa with special reference to pelvic inflammatory disease. Indian J Sex Transm Dis AIDS 2013;34(1):64-6.

[4] Spencer THI, Umeh PO, Irokanulo E, et al. Bacterial isolates associated with pelvic inflammatory disease among female patients attending some hospitals in Abuja, Nigeria. Afr J Infect Dis 2014;8(1):9-13. 
[5] Prasad JH, Abraham S, Kurz KM, et al. Reproductive tract infections among young married women in Tamil Nadu, India. Int Fam Plan Perspect 2005;31(2):73-82.

[6] Simms I, Warburton F, Westrom L. Diagnosis of pelvic inflammatory disease: time for a rethink. Sex Transm Infect 2003;79(6):491-4.

[7] Prathiba G, Pushpa J, Innocent DJP, et al. Prevalence of Chlamydia trachomatis infection in women in Chennai, India. Annals of Bio Research 2010;1(1):76-81.

[8] Chacko MR, Lovchik JC. Chlamydia trachomatis infection in sexually active adolescents: prevalence and risk factors. Pediatrics 1984;73(6):836-40.
[9] Machado MSC, Gomes ILC, Santana IU, et al. Prevalence of cervical Chlamydia trachomatis infection in sexually active adolescents from Salvador, Brazil. Braz J Infect Dis 2012;16(2):188-91.

[10] Gogate A, Brabin L, Nicholas S, et al. Risk factors for laparoscopically confirmed pelvic inflammatory disease: findings from Mumbai (Bombay), India. Sex Transm Infect 1998;74(6):426-32.

[11] Eggert J, Sundquist K, van Vuuren C, et al. The clinical diagnosis of pelvic inflammatory disease-reuse of electronic medical record data from 189 patients visiting a Swedish university hospital emergency department. BMC Womens Health 2006;6:16. 